\title{
Estudo De Mapeamento Sistemático Sobre As Tendências E Desafios Do Blockchain
}

\section{Systematic Mapping Study On Trends And Challenges Of Blockchain}

\author{
Juliandson Estanislau Ferreira ${ }^{1}$, Filipe Gutemberg Costa Pinto ${ }^{1}$, Simone Cristiane dos Santos ${ }^{1}$ \\ ${ }^{1}$ Universidade Federal de Pernambuco, UFPE, Brasil \\ Correspondência: Juliandson Estanislau Ferreira. Endereço: A. Prof. Moraes Rêgo, 1235, Cidade Universitária, \\ Recife, PE, CEP 50670-901 E-mail: estanislau18@gmail.com
}

Recebido: 30 de novembro de 2017 Aceito: 15 de dezembro de 2017 Publicado: 31 de dezembro de 2017

DOI: http://dx.doi.org/10.21714/1679-18272017v15Ed.p108-117

\begin{abstract}
Resumo
Blockchain é uma tecnologia de gerenciamento de dados cujo funcionamento consiste em transações descentralizadas, sendo desenvolvida para a criptomoeda Bitcoin no contexto de pagamento digital. O interesse na tecnologia blockchain tem aumentado desde que a ideia foi cunhada em 2008. As razões para o interesse na tecnologia blockchain são seus atributos centrais que fornecem segurança, anonimato e integridade de dados sem qualquer interferência de terceiros no controle das transações. Até agora, o blockchain tem atraído bastante atenção da indústria de serviços financeiros, mas a tecnologia pode ser adaptada para qualquer indústria onde seja necessário registrar, confirmar e transferir qualquer tipo de contrato ou propriedade. Nesta pesquisa, realizamos um mapeamento sistemático para estudar como essa tecnologia tem sido utilizada pelas corporações. O objetivo é compreender os principais recursos do blockchain, as novas aplicações que surgiram, impactos, vantagens e desafios. Foram extraídos 21 artigos primários de bases de dados científicas. Os resultados mostram potencial disruptivo da tecnologia, como ela vai mudar a nossa economia e como ela foi adaptada para muitas indústrias.
\end{abstract}

Palavras-chave: Blockchain, Inovação, Tendências, Tecnologia da Informação, Mapeamento Sistemático da Literatura.

\begin{abstract}
Blockchain is a data management technology whose operation consists of decentralized transactions being developed for Bitcoin cryptocurrency. The interest in blockchain technology has been increasing since the idea was issued in 2008. The reasons for the interest in blockchain technology are its core attributes that provide security, anonymity, and data integrity without any third-party interference in transaction control. So far, the financial services industry had taken a special attraction of the blockchain technology, however, any industry that has the need to register, confirm and transfer any type of contract or property can apply its technology. In this research, we have conducted a systematic mapping to study how this technology has been used by the corporations. The objective is to understand the main blockchain features, the new applications that have emerged, impacts, advantages and challenges. We have extracted 21 primary papers from scientific databases. The results show disruptive potential of the technology, how it will change our economy and how it has been adapted for many industries.
\end{abstract}

Keywords: Blockchain, Innovation, Trends, Information Technology, Systematic Literature Mapping.

Esta obra está licenciada sob uma Licença Creative Commons Attribution 3.0

\section{Introdução}

Blockchain é um banco de dados distribuído, online, público e que pode ser atualizado por qualquer nó participante da rede peer-to-peer ( $\mathrm{P} 2 \mathrm{P}$ ) baseado no consenso entre eles e assegurado por um algoritmo de uma prova de trabalho (Proof-of-Work), que tem o objetivo principal de dissuadir ataques cibernéticos (KYPRIOTAKI, ZAMANI; GIAGLIS, 2015). Utiliza-se de técnicas de criptografia para que cada participante possa manipular o ledger (livro digital onde informações são registradas regularmente) de forma segura e sem a 
necessidade de uma autoridade central. Uma vez que um bloco é adicionado ao blockchain é extremamente difícil alterar ou remover. Até agora, o blockchain tem atraído bastante atenção da indústria de serviços financeiros, mas a tecnologia pode ser adaptada para qualquer indústria onde seja necessário registrar, confirmar e transferir qualquer tipo de contrato ou propriedade. Blockchain é uma tecnologia tão disruptiva quanto a máquina a vapor e a eletricidade foram no século XX e espera-se que este novo paradigma seja capaz de modificar profundamente a maneira como a sociedade e a economia funcionam (UMALKAR, MACNEIL; LIGHT).

Diante deste contexto, este trabalho visa, por meio de uma investigação sistemática, identificar como o Blockchain está sendo usado para criar soluções inovadoras nas diversas indústrias existentes. Elencando os principais desafios, tendências e impactos. Este artigo está estruturado em sete seções. Nas seções 1 e 2 são apresentados a motivação e o referencial teórico para o entendimento e análise dos resultados da pesquisa. A seção 3 descreve a metodologia utilizada. A seção 4 descreve as etapas da pesquisa, contemplando o planejamento da pesquisa, execução e análise dos dados, respectivamente. A seção 5 apresenta as conclusões e considerações finais do trabalho. Finalmente, a seção 6 e 7 apresentam as referências e um apêndice contendo os artigos utilizados na revisão sistemática.

\section{Contextualizando o Blockchain}

O mundo está passando rapidamente por uma mudança fundamental: está cada dia mais sendo impulsionado pelos dados. E esta transformação ocorre em todos os sistemas sociais através de diversos serviços que englobam tráfego, saúde, governo, logística, marketing, segurança entre outros (SHRIER, WU; PENTLAND, 2016). No entanto, problemas como vazamentos de informação por parte dos provedores desses serviços requerem que novos modelos de segurança sejam criados. Uma das tecnologias que surgiram recentemente e com grande potencial para atender a esta necessidade tem-se o blockchain.

O blockchain consiste em uma cadeia cronologicamente ordenada de blocos protegidos pela resolução de Proofof-Work. $\mathrm{O}$ encadeamento é feito adicionando o hash do bloco anterior ao bloco atual. $\mathrm{O}$ alinhamento dos blocos de forma cronológica faz com que uma transação não possa ser alterada com antecedência sem alterar seu bloco e todos os blocos a seguir (AITZHAN; SVETINOVIC, 2016).

Um grande benefício do blockchain é que ele é uma nova forma de tecnologia de informação descentralizada que pode ser aplicável em muitas situações além da criptomoeda e ativos financeiros. A natureza descentralizada do blockchain torna-o uma tecnologia da igualdade, que pode ser usada expandir a liberdade, possibilidade, atualização, expressão, ideação, e a realização para todas as entidades no mundo, humano e máquina (SWAN, 2015).

O primeiro decentralized ledger para transações públicas foi desenvolvido por Satoshi Nakamoto (pseudônimo). No artigo Bitcoin: A Peer-to-Peer Electronic Cash System, de Nakamoto e publicado em novembro de 2008, o conceito foi introduzido pela primeira vez ao público (OBERHAUSER, 2014). O Bitcoin não foi o primeiro método de pagamento digital que foi desenvolvido, mas foi o primeiro que resolveu o double-spending (resultado de gastar algum dinheiro mais de uma vez) de forma descentralizada. Alguns exemplos de abordagens anteriores foram NetCash (1993), NetCents (1998) e uma patente com o nome de Method of electronic payments that prevents double-spending (1998). A abordagem apresentada no documento Bitcoin cria um ledger público descentralizado, denominado blockchain (OBERHAUSER, 2014).

\section{Metodologia}

A compreensão sobre os impactos, vantagens e desvantagens do blockchain se fez necessária por se tratar de uma abordagem relativamente nova em relação às tecnologias descentralizadas. Buscando conhecer esses e outros aspectos, o método escolhido para atingir o objetivo deste estudo foi o mapeamento sistemático da literatura. Este método permite a obtenção de uma visão ampla em uma área de pesquisa para determinar se existe evidência de pesquisa em um tópico e fornecer uma indicação da quantidade da evidência (KITCHENHAM; CHARTERS, 2007). A seguir, o protocolo do mapeamento é apresentado:

\subsection{Perguntas de pesquisa}

A pergunta condutora deste estudo foi: Qual o panorama do uso do blockchain nas empresas?

Com isso, as definições das perguntas específicas foram extraídas da pergunta condutora, e por possuírem um escopo reduzido, possibilitaram o alcance de respostas mais concretas. Portanto, as perguntas específicas formuladas foram:

\section{Q1: Quais são as principais características do blockchain?}



Q2: Como a tecnologia blockchain está sendo utilizada para inovar?
Q3: Quais os impactos que a tecnologia blockchain tem trazido para as empresas?
Q4: Quais as vantagens e desvantagens do blockchain?

\subsection{Busca e seleção dos estudos}

Para responder todas as perguntas de pesquisa, partiu-se para a estruturação da string de busca que seria utilizada nas fontes automáticas. Sua composição deu-se inicialmente a partir de termos relacionados às perguntas de pesquisa e levantamento em fontes relacionadas, conforme orienta Kitchenham e Charters (2007) e posterior validação do professor consultor. Desse modo, a string ficou da seguinte forma:

Tabela 1: String de Busca utilizada na pesquisa dos estudos

(blockchain OR "decentralized technology" OR "distributed ledger") AND
(innovation OR trends OR "business model" OR services) AND (perks OR
advantages) AND
(challenges OR disadvantages OR problems OR issues) AND
("information systems" OR "IT" OR "information technology")

Fonte: elaborados pelos autores (2017)

A busca automática foi realizada aplicando a string nas seguintes bibliotecas digitais e engenhos de busca, considerando os seguintes campos durante a busca: título, palavra-chave e resumo: Scopus, Science Direct, SpringerLink, IEEE Xplore e ACM Portal. Esta pesquisa teve início a partir de estudos provenientes de pesquisa ad-hoc no portal de periódicos da Capes, que serviram como base norteadora para a construção dos conceitos fundamentais sobre o blockchain e orientaram a formulação da String de busca. Por se tratar de um assunto complexo e relativamente novo, concluiu-se ser necessário uma maior compreensão do assunto e, posteriormente, partir para os meios formais de elaboração do mapeamento sistemático. Estes artigos iniciais foram categorizados como "Artigos Indicados".

Após a realização da busca, cada estudo teve seu título, palavras-chave e resumo avaliados, deixando de lado estudos que claramente fugiam do escopo do mapeamento, dentro do período imediatamente após o lançamento deste termo. Assim, para excluir os estudos no mapeamento foram considerados os seguintes critérios:

- O estudo estava indisponível para visualização;

- O estudo era duplicado;

- O estudo era um Slideshow;

- O estudo não estava no idioma inglês;

- O estudo não se encontrava entre o período de 2009 a $2016^{\mathrm{i}}$.

Durante a criação do protocolo do mapeamento também é importante avaliar os critérios de qualidade para os artigos que serão analisados. Eles ajudam, por exemplo, a orientar a interpretação dos resultados e determinar a força das inferências. Portanto, os critérios definidos para este mapeamento foram:

- Clareza do conteúdo;

- Ligação com o tema central;

- Proximidade do estudo em relação às questões de pesquisa.

\subsection{Extração dos dados}

De acordo com Kitchenham e Charters (2007), a extração dos dados tem por objetivo desenvolver formas de registrar com precisão as informações obtidas através dos estudos. Portanto, no presente trabalho, a extração de dados se deu através da criação de um formulário cujas informações eram registradas numa planilha eletrônica. O conteúdo extraído foram os metadados (itens relacionados ao título, ano de publicação, fonte e autores) e os fragmentos importantes que indicavam a resposta para cada uma das perguntas. Durante esta etapa, as perguntas específicas foram divididas entre os 4 pesquisadores e a partir de então, o estudo foi executado em três fases: análise do títtulo e resumo, análise da introdução e conclusão e, por fim, a extração de dados.

\section{Resultados}

A execução da busca automática retornou 199 resultados oriundos das ferramentas de busca onde 9 foram provenientes do Scopus, 108 do Science Direct, 33 do SpringerLink, 49 do IEEE e 0 do ACM. Além disso, 
durante a execução da busca manual, foram adicionados os 5 artigos iniciais aos indicados, estabelecendo o total de 204 artigos após a primeira etapa de seleção dos estudos para o mapeamento.

Após o retorno dos primeiros números, foram aplicados nos estudos o primeiro filtro, denominado Filtro 1 e que se constitui em analisar o título e o resumo, e os critérios de exclusão. Ao término desta etapa, 31 estudos foram selecionados. Em seguida, o segundo filtro, caracterizado por verificar a introdução e a conclusão, foi aplicado. No entanto, este filtro foi utilizado apenas nos estudos em que não era possível decidir se eram válidos ou não usando os critérios da etapa anterior. Com isso, o número alcançado foi de 21 estudos. Na etapa seguinte foram verificados se os estudos atendiam aos critérios de qualidade. No Quadro 1, é possível observar de forma resumida o número de estudos por fonte após cada etapa da busca.

Quadro 1: Distribuição dos resultados de acordo com as fontes de pesquisa

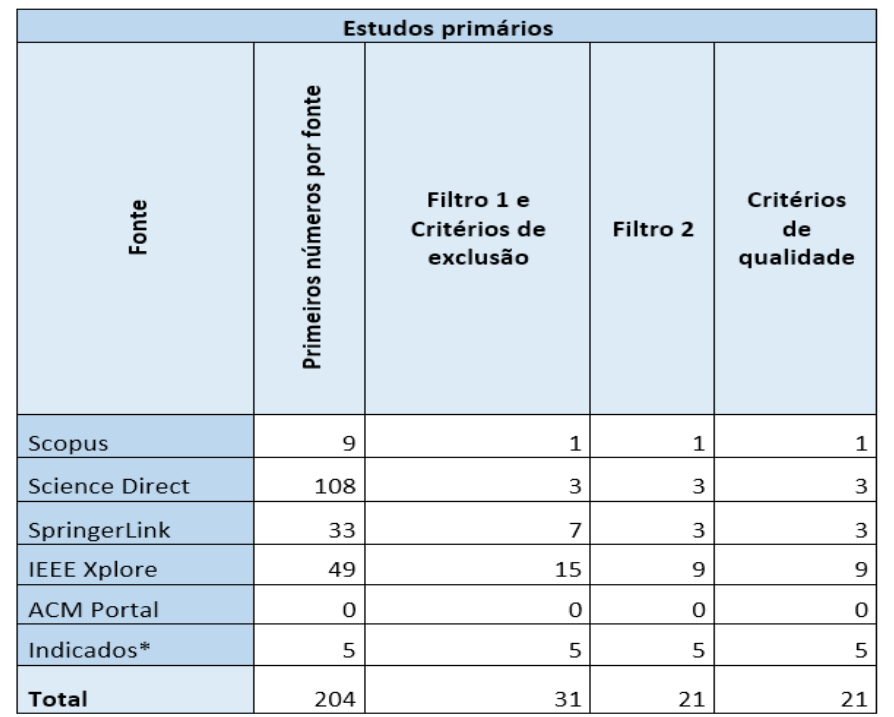

Fonte: Análise realizada em novembro de 2016.

Tabela 2: Distribuição da frequência dos estudos nas fontes de pesquisa

\begin{tabular}{lrr}
\hline Fonte de pesquisa & Número & Frequência (\%) \\
\hline Scopus & 1 & 5 \\
Science Direct & 3 & 14 \\
SpringerLink & 3 & 14 \\
IEEE Xplore & 9 & 43 \\
ACM Portal & 0 & 0 \\
Indicados & 5 & 24 \\
Total & $\mathbf{2 1}$ & $\mathbf{1 0 0}$ \\
\hline
\end{tabular}

Fonte: Análise realizada em novembro de 2016.

Gráfico 1: Distribuição dos estudos através dos anos 


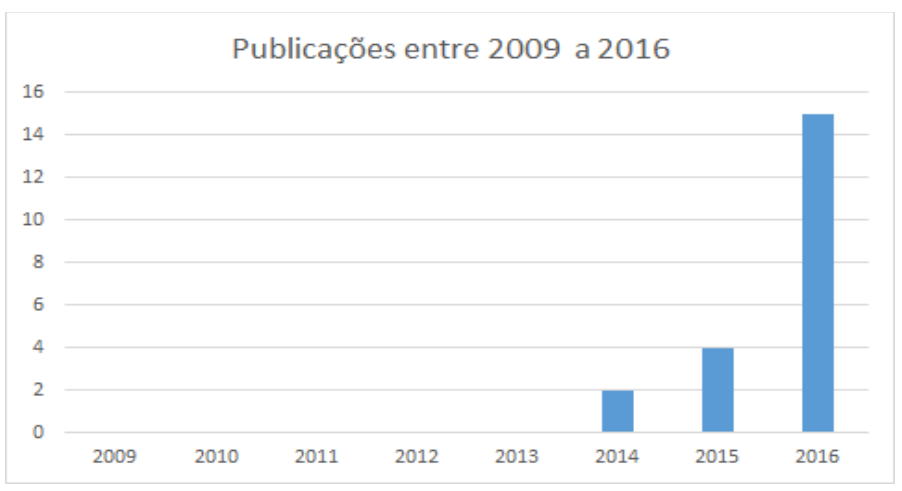

Fonte: Análise realizada em novembro de 2016.

Porém, nenhum dos estudos foram descartados, permanecendo o mesmo número de artigos e por fim, os 21 estudos foram considerados como relevantes para a extração e análise dos dados, sendo 16 provenientes da busca automática e 5 da pesquisa ad-hoc. Na Tabela 2, é possível conferir a distribuição da frequência dos estudos nas fontes de pesquisa. Já no Gráfico 1, é exibida a distribuição dos estudos através dos anos.

Com base nos dados expostos na Tabela 2 acima, o IEEE representa a fonte com maior número de estudos. Além disso, é possível verificar que o ano de 2016 foi um dos anos com maior número de publicações, demonstrando o aumento no interesse de pesquisadores pelo blockchain, que através dos números obtidos na própria análise deste trabalho, apresenta-se como uma tecnologia bastante recente. No Gráfico 2, observa-se a distribuição dos estudos por fonte com o número obtido após a realização de todas as etapas de seleção.

Gráfico 2: Estudos por fonte após a conclusão da seleção

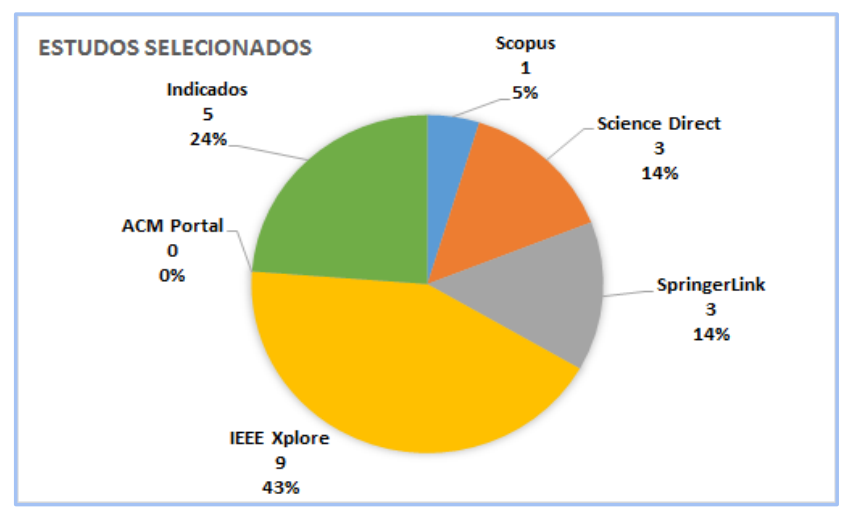

Fonte: Análise realizada em novembro de 2016

\subsection{Principais características do blockchain}

Buscando conhecer o funcionamento e os principais fatores que fazem do blockchain uma das tecnologias mais promissoras para os próximos anos, esta questão apresenta, através dos estudos selecionados, as suas principais características. Assim, os estudos EP005, EP006, EP007, EP009, EP020 e EP021 trazem o conceito de blockchain como uma cadeia cronologicamente ordenada de blocos protegidos por um processo de resolução de uma prova de trabalho (Proof-of-work). Segundo os trabalhos, o encadeamento é feito adicionando o hash de um bloco anterior ao bloco atual. Os blocos aninhados consecutivos garantem que as transações sejam enviadas em ordem cronológica, portanto, uma transação não pode ser alterada com antecedência sem alterar seu bloco e todos os blocos a seguir. O blockchain torna inviável o ataque de duplicação de gasto, a menos que o invasor controle mais de $51 \%$ do poder computacional da rede inteira.

Além disso, para adicionar um novo bloco à cadeia os seguintes conceitos são aplicados:

- Miners: De acordo com o EP003 um miner é um computador ligado à internet e que executa os cálculos necessários para verificar cada transação. Os miners efetivamente votam na legitimidade de cada transação como parte do processo de mineração através do timestamping de cada transação e verificam que ninguém tem realizado duplo gasto de dinheiro antes.

- Proof-of-Work: quebra-cabeça caro para resolver, mas que, sabendo todas as entradas, pode-se trapacear 
para verificar. Para gerar um bloco, um nó coleta transações pendentes, realiza o hash em cada uma e em seguida, juntamente com outros dados, iterativamente realiza o hash sobre este conjunto de dados até resultar em um hash menor ou igual a um alvo (target) predefinido. Target é um valor de hash que serve como um limite, abaixo do qual um cabeçalho de bloco deve ser feito o hash para gerar um bloco.

O estudo EP004 por sua vez, destaca que o blockchain pode ser visualizado como um termo geral para esquemas técnicos, semelhantes ao NoSQL, e pode ser realizado por muitos tipos de linguagens de programação. Já o estudo EP014 destaca o blockchain como um armazenamento de dados para muitas aplicações diferentes, fornecendo várias funcionalidades, entre as três primitivas: recuperar, atualizar e adicionar. A utilização como armazenamento de dados é bastante explorada neste estudo.

\subsection{Uso da tecnologia blockchain para inovar}

Os principais objetivos dessa questão são a investigação sobre quais setores econômicos estão usando o blockchain e destacar as principais aplicações envolvendo a tecnologia. Dentre os artigos analisados as principais aplicações se concentram nas indústrias de serviços financeiros, alimentos, energia, saúde e governo. Sobre este tema o estudo EP016 comenta:

A tecnologia Blockchain pode criar aplicações de registros públicos descentralizados, como títulos de terra, votação ou registros criminais, registros privados, como testamentos e trusts, enquanto que provavelmente será atendida em registros médicos ou emissão de certificações em um futuro próximo. Uma infinidade de ideias inovadoras, baseadas na tecnologia blockchain, já floresceu.

O estudo EP004 apresenta uma série de preocupações no que diz respeito à segurança alimentar no mercado chinês e propõe um sistema baseado em Radio-Frequency IDentification (RFID) e blockchain para rastreamento de alimentos dentro da cadeia de suprimentos. Tal sistema ajudaria a garantir a segurança e qualidade dos alimentos e ao mesmo tempo reduzir as perdas no processo de logística. O estudo EP005 aponta diversos desafios relacionados à segurança e privacidade para implantação de Smart grids (SGs). Para solucionar tais problemas implementa-se um sistema de troca de energia descentralizado utilizando blockchain, multi-signatures e criptografia, permitindo que os participantes negociem anonimamente os preços da energia e realizem com segurança transações comerciais. Os estudos EP002, EP003 e EP018 enfatizam como a tecnologia está sendo adotada no setor de serviços financeiros assim como o impacto na estrutura organizacional. Várias startups já estão utilizando esta ferramenta para criar novos serviços e oferecer valor a seus clientes. A R3 Financial Services Group espera executar suas operações financeiras instantaneamente. Outro exemplo é a, Coinbase, uma empresa que criou cartões de crédito e débito com base no Bitcoin. Sobre a importância do blockchain nos serviços financeiros o estudo EP018 destaca:

O sucesso do blockchain tem feito empresas de serviços financeiros se mover rapidamente para utilizar os beneficios de blockchain e sistemas semelhantes dentro de suas empresas. As empresas de serviços financeiros veem isso como apropriando-se da tecnologia blockchain dentro de suas próprias transações de liquidação. Estas poderiam incluir itens como liquidação de remessas e liquidações de hipotecas.

O estudo EP014 comenta sobre os principais desafios relacionados à implantação de sistemas da Internet das Coisas (IoT - Internet of Things), pois os modelos de dados mais utilizados atualmente não são escaláveis o suficiente para comportar milhões de dispositivos e usuários conectados. Para lidar com esse desafio o estudo propõe um sistema composto por três principais componentes, o primeiro é um protocolo de gerenciamento para permitir uma interação segura entre os usuários, o segundo um banco de dados descentralizado para que eles se comuniquem sem a necessidade de um intermediário e o por último um serviço de mensagens. $\mathrm{O}$ estudo ainda provê exemplos sobre como o sistema poderia ser utilizado em vários setores. São eles:

- Assistência Médica: O sistema permite que os profissionais de saúde compartilhem os registros de saúde eletrônicos diretamente entre si e evite a necessidade de troca de informações e formulários de autorização de papel.

- Cidades Inteligentes: A fim de tornar realidade a existência de cidades inteligentes, será fundamental ter modelos de compartilhamento de dados mais flexíveis e escaláveis para permitir o compartilhamento entre organizações. O modelo permite tal compartilhamento em pequena e grande escalas.

- Veículos Autônomos: Carros autônomos vão exigir comunicação de dados com outros carros ao redor, bem como com gerenciamento de tráfego. O sistema permite que carros autônomos decidam o que, quando e com quem seus dados devem ser compartilhados. 


\subsection{Os impactos que a tecnologia blockchain tem trazido para as empresas}

Esta questão visa analisar os impactos que a tecnologia blockchain tem trazido para as empresas e como essas empresas podem causar alguma transformação na sociedade. O estudo EP007 ao enfatizar o recente interesse de stakeholders através de uma ampla gama de indústrias como: finanças, assistência médica, utilitários, imobiliária e o setor governamental.

No caso da assistência médica, por exemplo, sempre que os registros de saúde de alguém são acessados, esse evento é registrado na cadeia de blocos, juntamente com as informações que foram alteradas ou adicionadas. Dessa forma, a informação permanece segura e inviolável, ninguém pode mudá-lo sem deixar vestígios.

Já no setor imobiliário o blockchain pode ocasionar disrupção ao agilizar o processo pois a maioria dos compradores e vendedores fazem uso de empresas de custódia e de títulos para verificação por terceiros - uma rede de segurança para garantir que ambas as partes mantenham o acordo, bem como reduzir o risco de fraude. Embora importante, esta verificação de terceiros tem um custo - normalmente 1 a $2 \%$ do valor total da propriedade e acrescenta tempo extra ao processo. Com blockchain, o intermediário (neste caso a empresa de custódia) poderia efetivamente ser cortada.

O estudo EP007 ainda aborda a utilização do blockchain em conjunto com IoT através dos Smart Contracts scripts auto executáveis que residem no blockchain, em relação a atualização de firmware de dispositivo IoT de um determinado fabricante. Neste caso, os blocos do blockchain poderiam conter a informação da última versão de firmware disponibilizada, e caso algum novo dispositivo entrasse na rede e o fabricante não pudesse fornecer o firmware, o dispositivo poderia buscar a informação em outros nós da rede blockchain e obter a atualização.

No entanto, uma vez que o blockchain ainda é uma tecnologia bastante recente, não foram encontrados nos demais estudos selecionados outras implementações existentes ou com ideias mais concretas que pudessem evidenciar outros impactos.

\subsection{Vantagens e desvantagens}

Esta questão tem como objetivo extrair as vantagens e desvantagens na utilização do blockchain em diversos contextos. Nos artigos estudados as seguintes vantagens do blockchain são recorrentes:

- Transparência nas transações;

- Auditabilidade;

- Criação de acordos sem a necessidade de um terceiro confiável;

- Anonimato;

- Banco de dados confiável.

O estudo EP004, discute que o blockchain poderia garantir a segurança de toda a rede utilizando um mecanismo de algoritmo matemático. Graças ao blockchain, todos os nós do sistema poderiam trocar seus dados de forma autônoma e segura no ambiente sem confiança.

O caso que trata do rastreamento de toda a cadeia de produção de alimentos, destaca as vantagens do blockchain, no sentido de como a tecnologia pode ajudar mercados agro-alimentares chineses para melhorar a sua qualidade e ao mesmo tempo, reduzir significativamente as perdas durante o processo de logística. Além disso, com base no sistema de blockchain, toda a informação do sector agro-alimentar na cadeia é aberta e transparente, assim a logística da empresa poderia implementar acompanhamento em tempo real dos produtos agro-alimentares, supervisores reguladores poderiam executar a gestão da rastreabilidade e investigação de responsabilidade por produtos defeituosos, obtendo informação completa dos produtos em toda a cadeia agro-alimentar, que são benéficas para estabelecer uma ambiente de mercados saudável.

O estudo EP007 pontua que com o blockchain, aquelas aplicações que antes só funcionavam através de um intermediário confiável, pode agora operar de uma forma descentralizada, sem a necessidade de uma autoridade central, e desempenhar a mesma funcionalidade com a mesma precisão. Isto era simplesmente impossível anteriormente.

Por outro lado, o estudo EP005 ressalta a importância do uso do blockchain quando quando se trata da preservação do anonimato do usuário. O trabalho mostra que um sistema descentralizado de comercialização de energia trustless ou semi-trustless poderia fornecer segurança e privacidade de identidade, ao confiar em técnicas criptográficas em vez de confiar em um terceiro.

O estudo EP021 também é categórico ao dizer que o blockchain possui várias vantagens. A primeira, é que 
atualmente ele existe como uma rede peer-to-peer que não tem um ponto único de falha. Se houver falha em qualquer nó, os outros nós continuarão a operar, mantendo a disponibilidade do sistema. Segundo, quase toda a documentação é digital e pode ser facilmente aplicada a muitas outras diferentes aplicações. Terceiro, todas as transações no blockchain são visíveis para todos os seus participantes, com o crescimento correspondente em auditabilidade e confiança. Quarto, as mudanças no blockchain são extremamente difíceis e em um caso muito raro caso viesse a ocorrer, isso seria visível para todos os usuários. Essas vantagens da tecnologia blockchain eliminará terceiros, diminuirá custos de transações, e causará transformações em muitas indústrias. Claramente, o blockchain é a coroa de jóias do protocolo Bitcoin.

No que tange às desvantagens da utilização do blockchain, as mais recorrentes são:

- Baixo nível de amadurecimento da tecnologia;

- Tamanho e largura de banda;

- Alta latência;

- Risco de Ataques;

- Desperdício de Recursos;

- Usabilidade;

- Versionamento.

Por ser uma tecnologia ainda recente, o sistema blockchain vem sendo constantemente aperfeiçoado, no entanto ainda apresenta limitações que em certos contextos podem se tornar custosas. Como apresentado no estudo EP007, por exemplo, atualmente a capacidade do blockchain é restrita a 7 transações por segundo devido à restrição do tamanho do bloco, enquanto que a VISA pode manipular mais que 47000 transações por segundo. Outro obstáculo é como lidar com o constante crescimento do tamanho do blockchain para questões como armazenamento e sincronização.

O estudo EP005 também mostra que devido às características descentralizadoras do blockchain, sua latência operacional tende a ser maior do que as soluções tradicionais centralizadas, no entanto isto pode ser compensado com a adição de um maior poder computacional.

Uma característica positiva do blockchain em relação às soluções centralizadas quanto à preservação da autenticidade dos dados, é que não adianta adulterar apenas a base de dados de um nó do blockchain, pois isto não terá efeito nos dados dos outros nós da rede, a menos que este detenha o controle de mais que $51 \%$ de todo o sistema de uma só vez.

Por fim, questões relacionadas ao desperdício de recursos, usabilidade e problemas de versionamento são tratadas no estudo EP019. Minerar Bitcoin desperdiça uma grande quantidade de energia (\$15milhões/dia). O desperdício em Bitcoin é causado pelo esforço em solucionar seu Proof-of-Work. A questão do desperdício de recursos precisa ser resolvida para que haja maior eficiência de mineração no blockchain. Junto a isto, a API do Bitcoin para desenvolvedores ainda é difícil de usar. Há a necessidade de que seja desenvolvido uma API mais amigável para o blockchain.

Com relação aos problemas de versionamento, quanto menor for uma cadeia de blockchain, maior será o risco de um ataque $51 \%$. Isso se torna ainda maior quando as cadeias são divididas por motivos administrativos ou questões de versionamento.

\subsection{Ameaças à Validade}

A primeira ameaça à validade diz respeito à estratégia de busca. Por causa da predominante utilização de busca automatizada, com exceção do portal de periódicos da CAPES, estudos relevantes podem não ter sido incluídos no conjunto de estudos selecionados.

A relativamente baixa quantidade de estudos primários analisados e os artigos indicados são considerados ameaças, pois podem ter tido influência no sentido de tornar o trabalho involuntariamente tendencioso ou menos abrangente do que poderia ter sido.

\section{Considerações Finais}

Com base nos estudos analisados, podemos concluir que o blockchain trouxe novas formas de soluções para antigos problemas das organizações. Seu paradigma descentralizador, aberto e acessível permite que os dados ali contidos sejam altamente confiáveis, tendo grande relevância no registro de transações, rastreabilidade e comprovação de propriedade. 
A tecnologia ainda está em sua fase de desenvolvimento por isso a maior parte dos estudos encontrados se concentram no ano de 2016. Por ser uma tecnologia recente, possui ainda um baixo nível de maturação, o que pode comprometer seu uso em larga escala no sentido de substituir as soluções tradicionais no curto prazo. Apesar das desvantagens, entendemos que o blockchain é uma solução revolucionária e que cada dia atrai a atenção de novas comunidades de desenvolvedores, portanto, suas falhas tendem a ser mitigadas à medida que sua aplicação se tornar mais difundida.

\section{Referências}

AITZHAN, N. Z:; SVETINOVIC, D. Security and Privacy in Decentralized Energy Trading through MultiSignatures, Blockchain and Anonymous Messaging Streams. IEEE Transactions on Dependable and Secure Computing, Outubro 2016.

KITCHENHAM, B.; CHARTERS, S. Guidelines for performing Systematic Literature Reviews in Software Engineering. EBSE Technical Report, 2007.

KYPRIOTAKI, K. N.; ZAMANI, E. D.; GIAGLIS, G. M. From bitcoin to decentralized autonomous corporations: Extending the application scope of decentralized peer-to-peer networks and blockchains. ICEIS 2015 - 17th International Conference on Enterprise Information Systems, 2015. 284-290.

OBERHAUSER, A. Decentralized Public Ledger as Enabler for the Gift Economy at Scale, 2014.

SHRIER, D.; WU, W.; PENTLAND, A. Blockchain \& Infrastructure (Identity, Data Security). connection.mit.edu, 2016.

SWAN, M. Blockchain Thinking: The Brain as a DAC (Decentralized Autonomous Organization). Texas Bitcoin Conference, 2015.

UMALKAR, M.; MACNEIL, A; LIGHT, D. What every CEO Should Know About Blockchain. accenture. Disponível em: <https://www.accenture.com/us-en/insight-outlook-executives-guide-to-blockchain\#blocksidebar>. Acesso em: 17 Setembro 2017.

\section{Apêndice - Estudos Primários Incluídos}

Quadro 2: Listagem dos estudos.

\begin{tabular}{|c|c|c|c|c|}
\hline Cód. & Título & Autor & Fonte & Ano \\
\hline EP001 & Cryptocurrencies and Business Ethics & $\begin{array}{l}\text { Claus Dierksmeier; Peter } \\
\text { Seele }\end{array}$ & Springer & 2016 \\
\hline EP002 & $\begin{array}{l}\text { The IoT electric business model: Using } \\
\text { blockchain technology for the internet of } \\
\text { things }\end{array}$ & Yu Zhang;Jiangtao Wen & Springer & 2016 \\
\hline EP003 & $\begin{array}{l}\text { The Ethics of Payments: Paper, Plastic, or } \\
\text { Bitcoin? }\end{array}$ & $\begin{array}{l}\text { James J. Angel; Douglas } \\
\text { McCabe }\end{array}$ & Springer & 2014 \\
\hline EP004 & $\begin{array}{l}\text { An Agri-food Supply Chain Traceability } \\
\text { System for China Based on RFID \& } \\
\text { Blockchain Technology }\end{array}$ & Feng Tian & IEEE & 2016 \\
\hline EP005 & $\begin{array}{l}\text { Security and Privacy in Decentralized } \\
\text { Energy Trading through Multi-signatures, } \\
\text { Blockchain and Anonymous Messaging } \\
\text { Streams }\end{array}$ & $\begin{array}{lr}\text { Nurzhan } & \text { Zhumabekuly } \\
\text { Aitzhan; } & \text { Davor } \\
\text { Svetinovic } & \end{array}$ & IEEE & 2016 \\
\hline EP006 & $\begin{array}{lll}\text { SoK: Research } & \text { Perspectives } & \text { and } \\
\text { Challenges for } & \text { Bitcoin } & \text { and } \\
\text { Cryptocurrencies } & & \end{array}$ & $\begin{array}{l}\text { Joseph Bonneau; Andrew } \\
\text { Miller; Jeremy Clark }\end{array}$ & IEEE & 2015 \\
\hline
\end{tabular}


Ferreira, J. E.; Pinto, F. G. C.; Santos, S. C. Revista Gestão.Org, v. 15, Edição Especial, 2017. p. 108-117

ISSN 1679-1827

http://www.revista.ufpe.br/gestaoorg

\begin{tabular}{|c|c|c|c|c|}
\hline EP007 & $\begin{array}{l}\text { Blockchains and Smart Contracts for the } \\
\text { Internet of Things }\end{array}$ & $\begin{array}{l}\text { Konstantinos Christidis; } \\
\text { Michael Devetsikiotis }\end{array}$ & IEEE & 2016 \\
\hline EP008 & $\begin{array}{l}\text { Interactive Incontestable Signature for } \\
\text { Transactions Confirmation in Bitcoin } \\
\text { Blockchain }\end{array}$ & $\begin{array}{l}\text { Yan Zhu; Ruiqi Guo; } \\
\text { Guohua Gan }\end{array}$ & IEEE & 2016 \\
\hline ЕР009 & A System View of Financial Blockchains & $\begin{array}{l}\text { Wei-Tek Tsai; Robert } \\
\text { Blower; Yan Zhu }\end{array}$ & IEEE & 2016 \\
\hline EP010 & $\begin{array}{l}\text { Decentralizing Privacy: Using Blockchain } \\
\text { to Protect Personal Data }\end{array}$ & $\begin{array}{l}\text { Guy Zyskind; Oz Nathan; } \\
\text { Alex 'Sandy' Pentland }\end{array}$ & IEEE & 2015 \\
\hline EP011 & $\begin{array}{l}\text { The global brain and the emerging } \\
\text { economy of abundance: Mutualism, open } \\
\text { collaboration, exchange networks and the } \\
\text { automated commons }\end{array}$ & $\begin{array}{l}\text { Ben Goertzela; Ted } \\
\text { Goertzelb; Zarathustra } \\
\text { Goertzel }\end{array}$ & Science Direct & 2016 \\
\hline ЕР012 & $\begin{array}{l}\text { Bitcoin blockchain dynamics: The selfish- } \\
\text { mine strategy in the presence of } \\
\text { propagation delay }\end{array}$ & $\begin{array}{l}\text { J. Göbela, H.P. } \\
\text { Keelerc,A.E. Krzesinskib, } \\
\text { P.G. Taylorc }\end{array}$ & Science Direct & 2016 \\
\hline EP013 & $\begin{array}{l}\text { Anonymity and privacy: a guide for the } \\
\text { perplexed }\end{array}$ & Danny Bradbury & Science Direct & 2014 \\
\hline EP014 & World of Empowered IoT Users & $\begin{array}{lcr}\text { Sayed } & \text { Hadi Hashemi; } \\
\text { Faraz } & \text { Faghri; Paul } \\
\text { Rausch; Roy H Campbell }\end{array}$ & IEEE & 2016 \\
\hline EP015 & $\begin{array}{l}\text { A Dispute Arbitration Protocol Based on a } \\
\text { Peer-to-Peer Service Contract Management } \\
\text { Scheme }\end{array}$ & $\begin{array}{l}\text { Jun Zou; Yan Wang; } \\
\text { Mehmet A. Orgun }\end{array}$ & IEEE & 2016 \\
\hline EP016 & $\begin{array}{l}\text { From bitcoin to decentralized autonomous } \\
\text { corporations: Extending the application } \\
\text { scope of decentralized peer-to-peer } \\
\text { networks and blockchains }\end{array}$ & $\begin{array}{l}\text { Kypriotaki, K.N., Zamani, } \\
\text { E.D., Giaglis, G.M. }\end{array}$ & Scopus & 2015 \\
\hline EP017 & $\begin{array}{l}\text { How online platforms challenge traditional } \\
\text { views of the firm and the regulation of } \\
\text { market failures }\end{array}$ & Bertin Martens & Indicação & 2016 \\
\hline EP018 & Corporate governance and blockchains & David Yermack & Indicação & 2015 \\
\hline ЕР019 & $\begin{array}{l}\text { Where Is Current Research on Blockchain } \\
\text { Technology?-A Systematic Review }\end{array}$ & $\begin{array}{l}\text { Jesse Yli-Huumo, } \\
\text { Deokyoon Ko, Sujin } \\
\text { Choi, Sooyong Park, Kari } \\
\text { Smolander }\end{array}$ & Indicação & 2016 \\
\hline EP020 & $\begin{array}{l}\text { Trusting records: is Blockchain technology } \\
\text { the answer? }\end{array}$ & Lemieux, V L & Indicação & 2016 \\
\hline EP021 & $\begin{array}{l}\text { Blockchain and Its Coming Impact on } \\
\text { Financial Services }\end{array}$ & $\begin{array}{l}\text { Kurt Fanning, David P. } \\
\text { Centers }\end{array}$ & Indicação & 2016 \\
\hline
\end{tabular}

\footnotetext{
${ }^{\mathrm{i}}$ Não foi considerado para fins de estudo o ano de 2008 devido ao fato de a tecnologia ter vindo à tona no final daquele ano.
} 